\title{
Digital Presentation and Communication of Cultural Heritage in the Post-Pandemic Era
}

\author{
F. F. Wang ${ }^{1, *}$, J. Du ${ }^{1}$ \\ ${ }^{1}$ Dunhuang Academy, Dunhuang, Gansu, China - (ffwangdh, dujuan0312) @ 126.com
}

\begin{abstract}
KEY WORDS: Digital Twin, New Cultural Creation, Media Convergence, Value Communication, Reshaping of Communication Ecology.
\end{abstract}

\begin{abstract}
:
In the post-pandemic era, coronaviruses will forge a long-term coexistence with humanity, and the fight against the pandemic will become the normal. With international social isolation and limited physical space for communication during the COVID-19 pandemic, the digital communication of cultural heritage based on internet technology has not only proven the importance and necessity of cultural heritage digitalization, but will also greatly accelerate the digital transformation that has already begun. In the post-pandemic era, the accumulation of rich digital data on cultural heritage will form the basis for further cultural communication. However, the collection of digital data is not the final link in this digital chain. Effectively leveraging the advantages of the digital technology - which spreads rapidly and has a broad audience as well as vivid information presentation - to integrate and digitally process the existing and emerging cultural heritage data will continuously combine the cultural heritage with new communication technologies. We should use diversified media platforms to achieve further breakthroughs in the communication model, present the digital communication content of the cultural heritage showing the characteristics of the times, and form real and virtual interaction of cultural heritage in a converged media environment, in order to further ease interpersonal isolation and even space-time constraints brought about by the pandemic, reconstruct the communication ecology of cultural heritage, and inherit and utilize the precious wealth left to humanity by history.
\end{abstract}

\section{INTRODUCTION}

The COVID-19 pandemic suddenly swept the world, quickly reversing the world pattern. While bringing enormous economic and political impact, it has also caused severe damage to the global cultural ecology. In terms of cultural heritage, an article published by UNESCO on April 9, 2020, stated that due to the pandemic, $89 \%$ of world heritage sites worldwide have been partially or completely closed to visitors. As of March 15, 2021, among 167 countries that have world heritage sites, $37(22 \%)$ countries have completely closed their world heritage sites, 41 (28\%) countries are still opening theirs, and 84 countries (50\%) have closed some of their world heritage sites(UNESCO, 2021). Due to travel bans, entry controls and import \& export restrictions, cultural exchanges among various countries have fallen sharply, and multicultural experience has been difficult to achieve. Interpersonal isolation measures have led to the closure of cultural venues and facilities and the cancellation of cultural activities, making it difficult for people to come into contact with culture, thus posing a huge challenge to cultural communication and access. Under the shadow of the COVID-19 pandemic, those who had been isolated anticipated access to comfort and relief brought by culture even more.

While the state of emergency caused by the outbreak of COVID-19 has made it difficult for the spread of cultural heritage to continue moving along its original trajectory, the demand for access to cultural heritage has transformed, and digital communication has given cultural heritage unexpected vitality in its development. During the battle against the pandemic, cultural heritage communication based on internet and digital media-based technology thrived, and various cultural institutions and museums have launched online exhibitions based on their local conditions. This has not only eased the tension caused by the physical space barriers of international communication(Yang, 2020), but also become the most unique and modern cultural landscape amidst international social isolation.

The purpose of the paper is to explore the sustainable development of cultural heritage communication in the postpandemic era and propose a new plan for achieving the integrated development of content, information, service and social interaction through the use of digital resources of cultural heritage installed with the three-dimensional communication matrix in the era of media convergence, thus providing effective countermeasures for achieving content production and value dissemination, overcoming special conditions faced by the recovery and development of cultural heritage undertakings after the pandemic and enabling people to maintain social links, acquire a sense of group belonging and eliminate interpersonal difficulties in the post-pandemic era.

\section{RESEARCH BACKGROUND}

\subsection{Shift from the digitization of cultural relics to the digital twin of cultural heritage}

In the long history of human civilization, a large number of precious cultural relics shine like pearls. Natural changes and human activity trajectories are constantly affecting their existence and development. Cultural relics inevitably suffer the erosion of time and damage from human activity. As early as the 1970s, foreign cultural workers began to explore the use of photography, video and other methods to preserve cultural heritage-related information. In China, the Dunhuang Academy began its digital conception of the Mogao Grottoes, a world cultural heritage site, in the late 1980s, and launched the "Digital Dunhuang" project at the end of the 20th century. At present, digital data collection of over 200 grottoes in Dunhuang has been completed. In 2016, the Dunhuang Academy released the "Digital Dunhuang" resource database platform(Dunhuang 
Academy, 2016) to the world, using modern digital technology to photograph, scan, obtain and store information on cultural relics in the Dunhuang Grottoes. It has now established a diversified and integrated digital Dunhuang database, a digital asset management system and a digital resource permanent preservation system. While achieving permanent preservation of Dunhuang cultural and artistic resources, it also provides unlimited possibilities for academic research and diversified use.

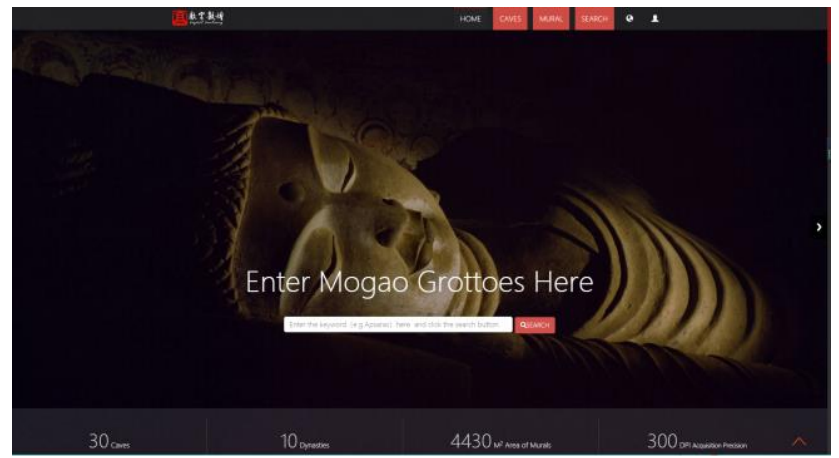

Figure 1. Digital Dunhuang resource database platform.

With the continuous development of technology, the digitization of cultural heritage is being constantly adjusted to adapt to changes of the times. With cultural heritage, China has shifted from the initial use of photography, video and other methods to preserve cultural heritage-related information to the use of digital technology to collect all-round data on cultural relics and is constantly developing towards the digital twin construction of cultural heritage, ushering the digital age for cultural heritage in China. The digital twin technology is not only conducive to the protection, inheritance, development and utilization of the physical entities of cultural heritage, but also provides brand new content and tools for digital presentation and communication of cultural heritage.

\subsection{Effective exploration of digital communication}

On March 5, 2021, Professor Su Bomin, Deputy Director of the Dunhuang Academy, stated in the first "Deputy Passageway" interview of the Fourth Session of the 13th National People's Congress that the Dunhuang Academy has used AR and digital twin technology to establish an intelligent tour of the Mogao Grottoes and a view of the cultural relics in the grottoes from outside, and has continuously innovated the communication channels and promotion models of Dunhuang culture through cloud exhibitions, media convergence communications and other methods. The digital twin of cultural heritage is the expression and description of the physical entity of cultural heritage. It is a virtual digital model that is consistent with the physical entity of cultural heritage in the real world(Qin and Zhang, 2018) and can be closely integrated with AR and VR technologies to provide a visual display and immersive experience of heritage sites to the public. It can also utilize digital communication media to expand the internet exhibition space on PC and mobile terminals under physical space constraints, allowing visitors to break through limitations of time and space and better appreciate the cultural heritage and its exhibition and to experience the cultural heritage in an immersive way, and therefore achieving immersion, experience and entertainment and bringing comfort and relief to people's minds.
The sudden outbreak of the COVID-19 pandemic has helped people to realize the importance of accelerating the digitalization of cultural heritage, and that digital presentation of cultural heritage has become the only way to manage the unknown physical space isolation and interpersonal isolation. Post-pandemic, the virtual panoramic roaming and immersive experience of cultural heritage supported by the digital twin technology shall become the norm for the field of public cultural services. However, this immersive panoramic roaming experience is only one aspect of the digital presentation of cultural heritage in the post-pandemic era - the digital twin of cultural heritage will promote further involvement of information technology in the protection, development and utilization of cultural heritage and provide solid digital support for exploring the value of cultural heritage, showing its meaning and achieving its diversified communication.

\section{METHODOLOGY}

This paper conducts comprehensive research through a combination of literature research and case study. It starts by comprehensively examining the frontier issues and theoretical research in the communication field in preliminary literature research, and then fully introduces relevant theories and methods of communication into the field of cultural heritage communication, analyzes communication cases with the communication theories and explores the use of frontier communication technological methods to solve problems related to the communication and development of cultural heritage in the post-pandemic era. In the case study, the paper systematically and structurally perceives the current situation of cultural heritage communication activities based on a full understanding of the current research directions, research focuses and main academic views of the academic community in the field through comprehensive research on the control field, content analysis, media analysis, audience analysis and effect analysis. Moreover, through research on the mining of cultural heritage communication value, the formulation of communication strategies, the planning of communication channels and the innovation of the forms of communication, the paper explores the internal driving forces and innovative expression modes of cultural heritage communication to promote the new development of cultural heritage communication in the post-pandemic era.

\section{KEY ISSUES AND COUNTERMEASURES}

\subsection{Key issues: Construction of the cultural heritage communication value system}

As a type of residual treasure of human history, cultural heritage has great historical, cultural, artistic and social value. The COVID-19 pandemic highlights the interrelated and interdependent relationship among people. This relationship is not only reflected in their interactions in the material world, but also in the emotional interactions in the spiritual world. If people use instrumental communications to monitor the physical world environment in order to smoothly and effectively implement all behaviors directly related to their own survival and development, then the communication of cultural heritage should be used to eliminate uncertainty in their emotional realm and the loneliness brought by space isolation, allowing them to better cope with the environment. The historical memory, cultural memory and social memory condensed by cultural heritage have awakened people in anxiety and despair in the 
face of crises, time and time again. What it brings to humans are emotional identity, group belonging and national resilience in the face of disasters. Cultural heritage brings a continuous confidence to people and allows people to experience the inheritance and changes of civilization. Cultural heritage will also continue to arouse people's love for culture and protect the cultural inheritance. It will continue to play an active role in helping humans build social connections, provide a sense of group belonging and conquer difficulties.

The construction of a cultural heritage communication value system in the post-pandemic era should break through the traditional communication model of the past which focused on the fragmentary value description of the external image of physical entities through visits, lectures and explanations, shifting towards a new communication model emphasizing immersive and inspiring development. In terms of content, the cultural heritage communication value system should excavate the philosophical thoughts, humanistic spirit and value concepts contained in cultural heritage, promote creative transformation and innovative development of cultural heritage values, reveal the human cultural spirit, cultural mind and cultural selfconfidence contained therein, and highlight the value integrity of cultural heritage. In the face of the global public health crisis, the value communication of world cultural heritage should stand at the height of a community with a shared future for humankind. Being complete proximity to the origin of cultural heritage value, it should be widely and deeply integrated into the daily life of the public, and use the discourse expressions favored by different audiences as well as care-filled presentation methods to establish a connection between human development and cultural heritage and to rebuild the unique scientific and cultural connotations of cultural heritage, in order to make cultural heritage the bridge between individuals and society and between history and reality, provide a more convenient channel for people to understand and be nourished by cultural heritage, as well as gain confidence and strength.

In addition, in the process of responding to and dealing with the COVID-19 pandemic in various countries and regions, problems and shortcomings that have been temporarily shelved in the development process of globalization have been fully exposed or even amplified, further intensifying the wave of "anti-globalization"(Wang, 2021). The tendency of "reverse globalization" has caused a lack of effective consensus and cooperation in various countries in their response to the pandemic, thus adding uncertainty to the already complicated fight against the pandemic. In such case, post-pandemic digital communication of cultural heritage appears to be quite important. The blending, promoting and co-existing multiethnic culture embodies internationality, inclusiveness, openness and innovation across time and space, helping cultural heritage become the best medium for exchange and dialogue between different races, beliefs and ethnic groups. On the other hand, as the crystallization of civilization formed in the continuous development and progress of mankind, the cultural heritage itself contains the spirit of inclusiveness, peace, progress and development. The excavation and dissemination of these cultural characteristics and values of the times are in line with the desire of people to restore pre-pandemic order and to forge a happy life in the post-pandemic era and help provide enlightenment and methods for reconstruction of global order, recovery of the world economy and the realization of common interests.

\subsection{New cultural creation drives the production of diversified digital content}

The COVID-19 pandemic has profoundly impacted living conditions and working methods. On the other hand, it has accelerated the digitalization of cultural heritage. While the offline cultural industry has sustained heavy losses, the online cultural industry has seen unprecedented prosperity, accelerating the germination of creative ideas, content production and the reshaping and transformation of communication channels for cultural heritage. During the pandemic, the digital scene that people experience as they live and work online will become a new direction for the future development of society. During the fight against the pandemic, China has continually explored new methods in public cultural services. How can cultural heritage leverage its precious value after the pandemic? How can it achieve sound development? Perhaps, new cultural creation can answer these questions.

In the field of cultural heritage, based on deep exploration of the time value of cultural heritage, abundant digital resources with new technologies and platforms are transformed into highquality and innovative communication content and digital culture through the excavation and combing of cultural values and the interpretation and activation of cultural IP. This makes the communication model more vivid and the presentation form of cultural heritage more three-dimensional and diversified. Finally, cultural heritage gradually becomes prominent under the integrated development of "culture + technology" and is fused into daily cultural life with innovative ways and impressive content. New business formats like new cultural creation continue to show the characteristics of the times, such as diversification, integration, innovation and leadership. New cultural business formats will continue to emerge with new innovative models, providing Chinese solutions for the development of the global digital cultural industry(Yang and Cui, 2020). Cultural heritage breaks through the traditional concept of content production, takes new cultural creation as the core development concept and cross-border integration as a new development model, and fully leverages digital methods to promote the construction of traditional cultural IP, using animation, movies and online videos to promote the creative transformation and innovative development of cultural heritage resources and aid in the inheritance and innovation of traditional culture.

In China, the cooperation between the Dunhuang Academy and Tencent is worth promoting. As representatives of cultural and technological communities, both enterprises will fully leverage their respective advantages, implement in-depth cooperation in cloud computing, smart tourism, games, animation, music and other modules, apply Dunhuang's cultural IP in film, music, animation, games and other fields, fully empower cultural heritage, promote cultural protection and exchanges on the Digital Silk Road, and continue to exhibit the characteristics of the times, such as diversity, integration, innovation and leadership. In the early days of the pandemic outbreak, the two enterprises achieved integrated development of internet cultural tourism via a miniApp named "Cloud Tour of Dunhuang". Later on, they successively launched popular features for the miniApp, such as "Dunhuang Animation Drama" and "Cloud Collection of Silk Scarf," allowing the miniApp connect over a billion users on Tencent's social matrix. Traffic to the miniApp has exceeded 39 million, creating a benchmark case for the digital cultural heritage and museum industry and is a sample model for the online experience of cultural heritage IP application. 


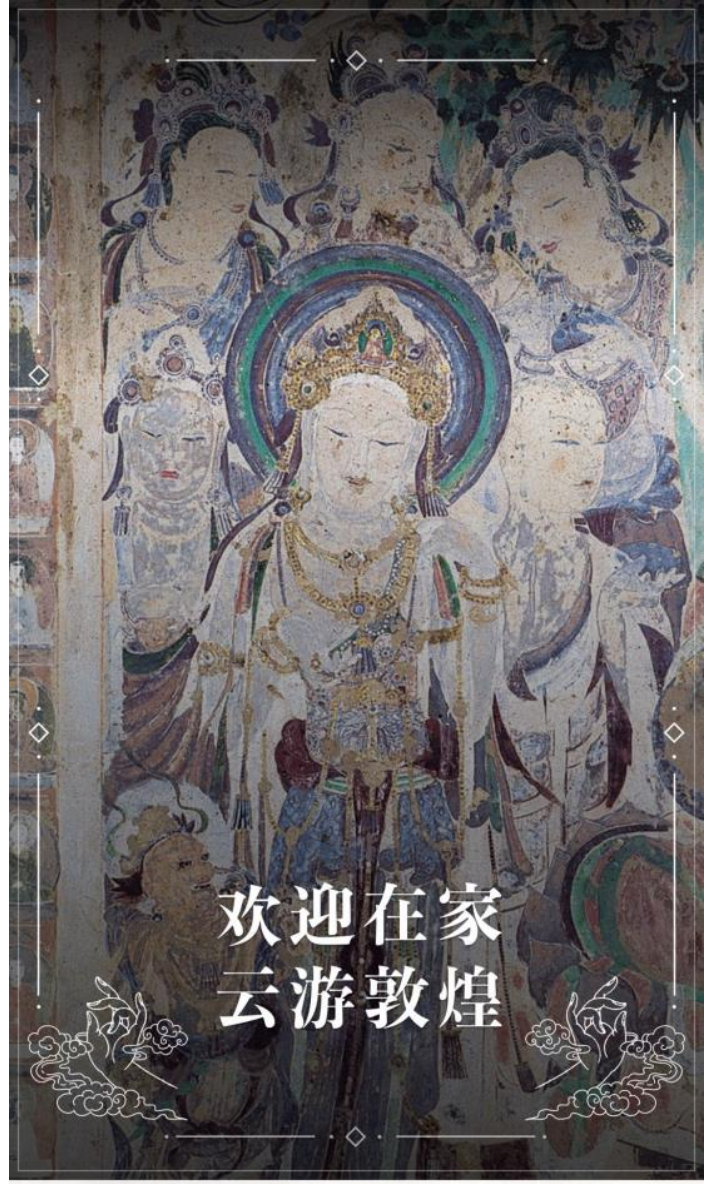

\section{长按本图} 即刻启程

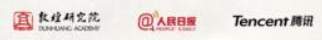

Figure 2. Cloud Tour of Dunhuang.

New cultural creation continually seeks a point of convergence between cultural heritage and new technology and provides multi-form content products that meet the requirements of the new communication pattern aimed at different target audiences by combining the communication advantages of the internet platform, in order to gain the development advantage of media convergence through high-quality content. On February 4, 2021, the Dunhuang Academy teamed up with Tencent and People's Daily New Media to launch the "Light Up the Mogao Grottoes" function on the WeChat version of the "Cloud Tour of Dunhuang" miniApp. For the first time, this new cultural creation program, which uses mobile digital technology and blockchain technology, reproduced a 1,000-year-old folk tradition of burning lamps in the Mogao Grottoes for the public during the 2021 Chinese New Year, using technology to display the magnificent scene of "the Milky Way hanging over the river." ancient cultural heritage continues to collide with new cultural creation, bringing a greater imagination space for the audience. With the support of technology and platforms, more young people can recognize, understand and come to love cultural heritage, becoming the disseminators and innovators of cultural heritage. Authors and innovators must not only tell the story of cultural heritage, but also inspire others to take initiative in exploring their curiosity in the story. Through the organic combination of traditional cultural resources and information technology, the new post-pandemic cultural creation model not only allows people to fully understand the historical value, cultural value, social value, technological value and artistic value of cultural resources, but also relieve the shortage of culture and entertainment experienced by those staying at home, distribute the pressure of tourist reception of cultural heritage sites, and effectively reduce the adverse effects of "explosive" post-pandemic tourism on cultural heritage sites.

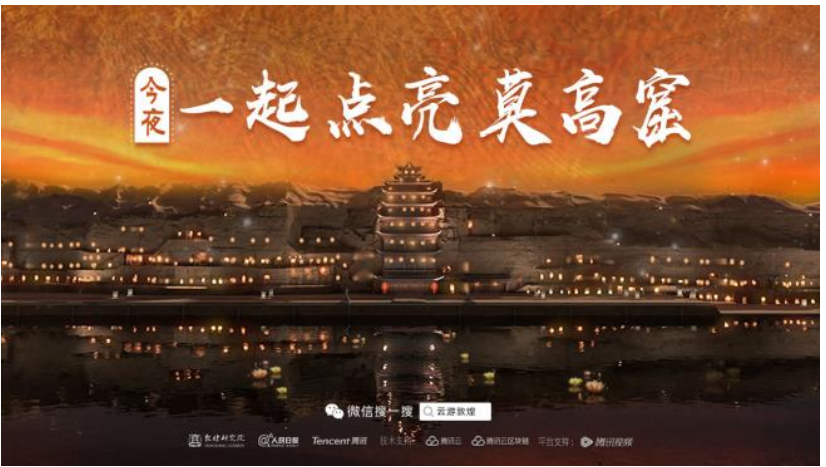

Figure 3. Light Up the Mogao Grottoes.

\subsection{Three-dimensional communication matrix under a high degree of media convergence}

In the modern communication environment, traditional communication media has lost its versatility, but new media technologies are developing rapidly and the number of mobile users is doubling. Naturally, traditional communication models cannot match the platform attributes of emerging media. The use of new technologies, new platforms and the advantages of the emerging media to innovate media communication modes to achieve the vertical and in-depth development of media convergence has become the trend of the times and the general trend. In the context of media convergence, full-course media that transcends boundaries of time and space, the holographic media that exceeds physical limitations, me media that breaks the boundaries of the subject and the full-effect media that overcomes the boundaries of functions all promote the integration of content, information, service and social interaction and achieve the interaction of reality with the virtual. Cultural heritage, equipped with cutting-edge technology, will acquire new opportunities in content production, value mining, communication form innovation and communication effect analysis. With the renewal of technology, the revival of cultural undertakings and the growing demand for public culture, the three-dimensional communication matrix under a high degree of media convergence is a new direction for the digital communication of cultural heritage, and more diverse and convenient communication forms provide a broad development space for the creation of cultural heritage communication products.

The move by cultural heritage institutions to establish a smart media convergence platform for internet and mobile businesses can achieve unified data, unified operations, unified back-end and unified management and create a new communication channel. On the one hand, it combines the latest science and technology with the value and connotation of cultural heritage and provides personalized display for different audience groups, so that the cultural heritage communication content can be spread out on the omni-media platform to achieve the effect of having the same voice and the same tone and resonating at the same frequency, to form a real and virtual interaction of cultural heritage in the media convergence environment, to achieve a wide range of audience training and to promote the integration of cultural heritage into daily life. This can effectively enhance 
the communication, guidance and influence of cultural heritage and increase cultural exchange and dialogue and cultural communication capabilities. On the other hand, cloud computing, big data and other technologies possessed by the media convergence platform can excavate new communication hotspot resources through data analysis, adjust communication strategies in time and spread cultural heritage. At the same time, cultural heritage has established an agenda and actively enters the mainstream communication system, not only improving the influence of cultural heritage communication, but also providing excellent cultural guidance and correct value orientation in an internet communication environment with massive amounts of information and homogeneous content, allowing cultural heritage to be integrated into contemporary life and earn the favor of more young people as well as reconstruct the cultural heritage communication format.

The digital presentation and communication of cultural heritage must fully adapt to the multi-device usage habits of users, and fully meet the diverse spiritual needs of different groups through multiple methods, such as short video, web animation, cloud live broadcast and VR technology. We should carefully create branding and diversified displays of outstanding cultural works, paying particular attention to avoid misunderstandings caused by cross-cultural differences. Of course, in order to establish a three-dimensional communication matrix of cultural heritage under a high degree of media convergence, it is necessary to enhance the building and operation of a team of talent and improve its hematopoietic capabilities, establish a composite omni-media professional talent team, and effectively operate the media convergence platform in an all-rounded, multi-angled and differentiated manner. It is important to arouse people's cultural memory and value identity through interactive communication-style value guidance.

\section{CONCLUSIONS}

Post-pandemic, the dissemination of cultural heritage should explore the content production and development model represented by new cultural creation based on data accumulation, centered on value system construction and modelled on converged media communication, in order to create new opportunities for the recovery and development of cultural heritage communication in the post-pandemic era. In the process of dissemination, communicators must continue to dig deep into the spiritual core contained in various themes of cultural heritage to create excellent communication products that encapsulate diverse perspectives and the spirit of the times. On the one hand, it is necessary to bring forth the new through the old, carry out extensive research in the selection and setting of communication themes, and deeply understand the general expectations and spiritual needs of the public at home and abroad for cultural heritage;on the other, we must broaden horizons and make extensive use of new media technologies to explore the structured and interesting presentation of cultural heritage content and value. On the premise of fully being close to the origin of cultural heritage value, it is necessary to tell warm, tasteful and inspiring stories about cultural heritage in ways the general public will enjoy, and present them with humanity.

\section{DISCUSSION}

Of course, when discussing digital presentation and communication of cultural heritage in the post-pandemic era, some objective issues must also be addressed. Affected by the global COVID-19 pandemic, the survival and development of some countries and people are still under threat. In these countries, most cultural heritage sites that rely on tourism revenue to maintain their operations are facing financial difficulty, and the digital transformation and development of cultural heritage cannot be achieved yet. In the post-pandemic era, countries first entering the recovery and development of their national economies will inevitably lead the development of cultural heritage through digital technology, and the information gap and wealth gap originally existing among different countries and regions caused by the difference in the capability of these countries and regions in acquiring, applying and innovating information and network technologies will further be polarized. In some areas where poverty has been exacerbated due to the pandemic, the digital gap and knowledge gap will become more prominent. Intercultural exchange and communication will inevitably be met with resistance by some countries and people out of prejudices caused by "politicization", and thus the international communication of cultural heritage will encounter many difficulties in the post-pandemic era, and the diversity of cultural expression forms will also be damaged.

In addition, the digital presentation and communication of cultural heritage require both integrity and innovation. The communication form and content of cultural heritage are fragmented in the context of media convergence, and the cultural experience in the virtual environment cannot be compared to that in the real environment at all, either, so the authenticity and integrity of the cultural heritage will be affected. The protection, preservation, display and communication of cultural heritage is an enormous systematic project. It not only involves archeology, museology, geology, informatics, materials science, communication and other multidisciplinary knowledge, but also requires the organic integration and crossover of the knowledge of various disciplines(Pan et al., 2020). In-depth research on cultural heritage requires gathering and cultivating of top cultural heritage talent through multi-disciplinary crossover and formation of a new mechanism that is conducive to the innovative transformation of major achievements as well as the in-depth integration of technology and culture. However, the shortage of talent in some cultural heritage institutions cannot be alleviated for a long time. On the other hand, content will become the key to truly defining the value of communication in the digital environment. The output of high-quality content translates to high intellectual and time costs. This requires that those engaged in the spread of cultural heritage to strictly control costs, innovatively excavate excellent traditional cultural elements in the content and form and inject the elements of the times into them, absorb the value provided by modern civilization to meet the needs of people in the new situation, and ensure the high quality, high standard and high taste of the communication content.

\section{REFERENCES}

Dunhuang Academy, 2016. "Digital Dunhuang" resource database platform, https://www.e-dunhuang.com(10 March 2021).

Pan, Z., Yuan, Q., Chen, S., Zhang, M., 2020. State of the art on the digital presen tation and in teraction of culture heritage. Journal of Zhejiang University(Science Edition), 47(3), 261-273. 
Qin, X., Zhang, X., 2018. Application of Digital Twin Technology in the Digital Construction of Material Cultural Heritage. Information and Documentation Services, 02, 103-111.

UNESCO, 2021. Introduction to the global map on the closure of World Heritage sites due to Covid-19 and analysis, https://en.unesco.org/covid19/cultureresponse/monitoringworld-heritage-site-closures (15 March 2021).

Wang, H., 2021. The Value and Trend of Construction of a Community with Shared Future for Mankind in the Post COVID-19 Era. International Communications, 48(2), 66-70.

Yang, X., Cui, L., 2020. Accelerate to stimulate new drivers for the development of the digital cultural industry under the situation of COVID-19 pandemic prevention and control. Social Scientist, 273, 132-136.

Yang, Y., 2020. Thoughts on the International Communication of Chinese Culture in the Post-pandemic Era. International Communications, 287, 14-17. 